\title{
О РОЛИ СОНДИРОВАНИЯ В ЗАДАЧАХ УПРАВЛЕНИЯ ПО НЕПОЛНЫМ ДАННЫМ. ВЫБОР МОМЕНТА НАБЛЮДЕНИЯ
}

R. TENNO. VAATLUSMOMENDI VALIKUST VAEGINFORMATSIOONIGA OPTIMAALJUHTIMISE OLESANDES

R. TENNO. OPTIMAL SELECTION OF THE OBSERVATION TIME IN THE CONTROL PROBLEM WITH UNKNOWN PARAMETERS

\section{(Представил Н. Алумяэ)}

Активное накопление информации характерно для задач оптимального управления по неполным данным особенно в начальный этап, когда управления являются наиболее сондирующими (агрессивными). Ввиду сложности задач в литературе нет оценок длительности интенсивного сондирования, кроме численных примеров (см. напр. $\left.\left[{ }^{1,2}\right]\right)$. Такие оценки могут быть получены в простых задачах с обозримой структурой. Они позволяют глубже понять ту роль, которую играет (или не играет) сондирование в оптимальном управлении. Рассмотрим одну из таких задач.

Пусть $M$ - математическое ожидание, $\lambda$ - любая константа, $N-$ натуральное число. Требуется найти пару $(\tau, \alpha)$, где $\tau-$ момент наблюдения $(1 \leqslant \tau<N), \alpha-$ стратегия управления с обратной связью вида $\alpha_{t}=\alpha\left(t, \xi_{\tau}\right), \tau \leqslant t$, такую, которая минимизирует функционал

$$
v^{\alpha}=M\left\{\sum_{t=1}^{\tau}\left(\Theta_{t}-\lambda\right)^{2}+\sum_{t=\tau+1}^{N}\left(\Theta_{t}-\lambda\right)^{2}\right\},
$$

если управляемая последовательность задана уравнением

$$
\Theta_{t}=\alpha_{t-1}^{\mathrm{T}} \beta+\varepsilon_{1}(t)
$$

и наблюдается

$$
\xi_{\tau}=\Theta_{\tau}+\varepsilon_{2}(\tau) .
$$

Здесь $\varepsilon_{1}(t), t=1, \ldots, N, \varepsilon_{2}(\tau)$ - независимые, центрированные гауссовы величины с дисперсиями $\sigma_{1}, \sigma_{2}$ соответственно, $\sigma_{1}+\sigma_{2}>0, \beta-$ вектор неизвестных параметров. Заданы гауссова оценка $a$ вектора $\beta$ и положительно определенная матрица ковариации $A$ оценки $a$.

Оценка момента наблюдения. В принятыч условиях прогноз неизвестных параметров (а также прогноз возмушений $\{\varepsilon(t)\}$ ) один и тот же для любых упреждений. Поэтому оптимальная стратегия строится следующим образом. С помощью среднего $a$ и ковариации $A$ априорного распределения выбирается управление на первом шаге $\alpha_{0}$ и фиксируется на данном уровне $\alpha_{t}=\alpha_{0}$ до момента поступления наблюдений $\tau$. C помощью наблюдения $\xi_{\tau}$ вычисляется новое управление $\alpha_{\tau}=\alpha\left(\tau, \xi_{\tau}\right)$, которое затем фиксируется $\alpha_{t}=\alpha_{\tau}$ до конца интервала управления. Такой выбор стратегии позволяет свести задачу (1)-(3) к двухшаговой с критерием оптимальности 


$$
v^{\alpha}=\tau S+(N-\tau) R
$$

Здесь

$$
S=\left(\alpha_{0}^{\mathrm{T}} a-\lambda\right)^{2}+\alpha_{0}^{\mathrm{T}} A \alpha_{0}+\sigma_{1}
$$

- риск действия,

$$
R=M \inf _{\alpha_{\tau}} M\left\{\left(\Theta_{\tau+1}-\lambda\right)^{2} / \xi_{\tau}\right\}
$$

- риск изучения. Согласно $\left[{ }^{3}\right]$ функцию $R(\alpha)$ можно записать в более удобном виде, с помощью решения $w(z)$ дифференциального уравнения

$$
\frac{d w}{d z}=\frac{2 i}{\sqrt{\pi}}-2 z w(z)
$$

от комплексного аргумента $z=x+i y$. Другими словами,

$$
R=\sigma_{1}+\lambda^{2} y \sqrt{\pi} \operatorname{Re} \omega(z),
$$

где (в слу́чае одномерного управления)

$$
\begin{gathered}
\sqrt{2} x=-\frac{a}{T}, \quad 2 y^{2}=\frac{A}{T^{2}}-1, \\
T=A \alpha_{\tau} / \sqrt{\sigma_{1}+\sigma_{2}+A \alpha_{\tau}^{2}} .
\end{gathered}
$$

Оптимальное управление на втором этапе $\alpha_{\tau}{ }^{*}$ такое же, как и в случае $N=2$, а на первом этапе $\alpha_{0}{ }^{*}$ и оптимальный момент наблюдения $\tau^{*}$ удовлетворяют трем условиям:

$$
\mathrm{S}=R, \quad \tau S_{\alpha}+(N-\tau) R_{\alpha}=0,
$$

матрица

$$
\tau S_{\alpha \alpha}+(N-\tau) R_{\alpha \alpha}
$$

неотрицательно определена. Здесь $S_{\alpha}, R_{\alpha}-$ первые, а $S_{\alpha \alpha}, R_{\alpha \alpha}-$ вторые производные от функции $S\left(\alpha_{0}\right), R\left(\alpha_{0}\right)$. Если управление одномерное $\|$ если для простоты отказаться от требования целочисленности $\tau$, то получим простую оценку

$$
\tau^{*}=\frac{R_{\alpha}}{R_{\alpha}-S_{\alpha}}\left(\alpha_{0}^{*}\right) N
$$

Риск действия и риск изучения изменяют управление в противоположном направлении. Поэтому

$$
\frac{S_{\alpha}}{R_{\alpha}}\left(\alpha_{0}^{*}\right) \leqslant 0
$$

В широких пределах изменения параметров $\left|R_{\alpha}\left(\alpha_{0}^{*}\right)\right| \ll\left|S_{\alpha}\left(\alpha_{0}^{*}\right)\right|$, тем самым $\tau^{*} \ll N$.

\section{ЛИ ТЕРА Т Р А}

1. MacRae. E. C. Ann. of Economic and Social Measurement, 1, № 4, 437-447 (1972).

2. Tse, E.. Bar-Shalom, Y. IEEE Trans. Autom. Contr.. 18, № 2, 109-177 (1973).

3. Тенно Р. Изв. АН ЭССР. Физ. Матем., 32, № 1, $11-18$ (1983). 